\title{
Analysis of Singular Stress Fields Along a Singular Line Near the Vertex in 3D Piezoelectric Bonded Joint Using a Conservative Integral
}

\author{
(In case of two-real singularities) \\ [Chonlada LUANGARPA, Hideo KOGUCHI]
}

\begin{abstract}
In the present study, singular stress fields in three-dimensional piezoelectric bonded joint with two-real singularities are investigated at a vertex and along a free edge (a singular line) of an interface. A conservative integral formulation is developed for calculating the intensity of singularity at a point located on the singular line of threedimensional piezoelectric bonded joint. The intensities of the singularities at several positions along the singular line of the interface are determined. The relationships between singular stress fields at the vertex and along the singular line are considered.
\end{abstract}

Keywords-stress singularity, bonded joint, conservative integral, piezoelectric material

\section{Introduction}

Piezoelectric materials have been frequently used in numerous engineering and technology products, e.g., sensors or actuators. Piezoelectric bonded joints have singularities created by discontinuities in material properties across interfaces. The stress singularities caused from mechanical or electric inputs may lead to fracture and failure, so the investigation of singular stresses in the piezoelectric bonded joints is important.

In the present study, singular stress fields in threedimensional piezoelectric bonded joints are investigated. The singular stress fields in three-dimensional bonded joints are more complicated than those in two-dimensional joints. In three-dimensional bonded joints, singular stresses are generated not only at the vertices but also along the free edges of the interface. Three-dimensional conservative integral developed by Luangarpa and Koguchi [1],[2] is extended to the intensity of singularity at a point located on the singular line (along a free edge) of three-dimensional piezoelectric bonded joint. Eigenanalysis formulated by a three-dimensional FEM (Islam and Koguchi [4]) is used for calculating orders of stress singularity, angular functions of displacements and stresses.

The intensities of singularity along the singular line of the bonded joints with one-real singularity were investigated by Luangarpa and Koguchi [2]. They proposed that the intensities of the singularities along the singular line could be described as a function of the distance from the vertex and the singular stress at the vertex. We are studying further

Chonlada LUANGARPA*, Hideo KOGUCHI**

Nagaoka University of Technology

1603-1 Kamitomioka-machi, Nagaoka, Niigata, Japan in case of multi-term of singularities. To our knowledge, no study in case of multi-term of singularities has been conducted. Hence, in this study, the piezoelectric bonded joint with multi-term of singularities is investigated; and the intensities of singularities at several positions along the singular line of the interface are examined.

\section{Analytical Formula}

A conservative integral along a singular line in threedimensional piezoelectric bonded joints is developed using Betti's reciprocal principle as follows:

$$
\oint_{S}\left(T_{i}^{\prime} u_{i}-T_{i} u_{i}^{\prime}\right) d s=0 \text {. }
$$

For any contour $S, T_{i}$ and $T_{i}(i=1,3)$ are tractions, and $u_{i}$ and $u_{i}^{\prime}$ are displacements of the singular and complementary fields, respectively. For piezoelectric material, additional terms related to electric properties are added; $T_{4}$ and $T_{4}$ are the electric displacement with the outward unit vector $\left(T_{4}={ }_{4 j} \hat{n}_{j}\right)$, and $u_{4}$ and $u_{4}^{\prime}$ are the electric potentials. This principle is extended to solve the three-dimensional bi-material model as shown in Fig. 1. Equation (1) is rewritten as an integral with respect to the closed area shown in Fig. 2 as follows:

$$
\sum_{j=0}^{3} \oint_{S_{j}}\left(T_{i}^{\prime} u_{i}-T_{i} u_{i}^{\prime}\right) d s=0 .
$$

The contour, $S$, is chosen as $S=S_{0}+S_{1}+S_{2}+S_{3}$, where $S_{2}$ and $S_{3}$ are on free-surfaces, then the traction is free on these surfaces so that

$$
\left.\left(T_{i} u_{i} \quad T_{i} u_{i}\right) d s+\underset{S_{1}}{\left(T_{i} u_{i}\right.} \quad T_{i} u_{i}\right) d s=0 .
$$

Modifying the form of traction to be $T_{i}={ }_{i j} \hat{n}_{j}$, where $\hat{n}_{j}$ is the outward unit vector to the closed surface, $S$. Let $\hat{n}_{j}$ be the unit vector in the reversed direction of $\hat{n}_{j}$, such that

$$
\left({ }_{i j} u_{i} \quad{ }_{i j} u_{i}\right) \hat{n}_{j} d s={ }_{S_{1}}\left({ }_{i j} u_{i} \quad{ }_{i j} u_{i}\right) \hat{n}_{j} d s .
$$

Equation (4) proves that the integral is area-independent.

The $H$-integral at the vertex in $3 \mathrm{D}$ dissimilar materials is defined as follows:

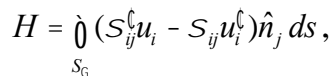

where $S$ is an arbitrary area enclosing the singular point (Fig. 3). 
The asymptotic stresses around the singular point in spherical coordinate system can be described as follows:

$$
{ }_{i j}(r, \quad, \quad)=\sum_{n=1}^{m} K_{n}\left(\frac{r}{L}\right)^{-n} f_{i j}^{(n)}(, \quad),
$$

where $r$ is the radial distance from the singular point, $m$ is the number of singularity term; $i, j=r, \theta, \phi$, and $\lambda, K$, and $f_{i j}$ are the order of the stress singularity, the intensity of singularity, and angular functions, respectively.

The displacement fields are given by

$$
u_{i}(r, \quad)=\sum_{n=1}^{m} K_{n}\left(\frac{r^{1} n}{L^{n}}\right) g_{i}^{(n)}(, \quad) .
$$

The electric displacement and electric potential can be written as follows:

$$
\begin{aligned}
&{ }_{4 j}(r, \quad)=\sum_{n=1}^{m} K_{n}\left(\frac{r}{L}\right)^{-n} f_{4 j}^{(n)}(,), \\
& u_{4}(r, \quad, \quad)=\sum_{n=1}^{m} K_{n}\left(\frac{r^{1} n}{L^{n}}\right) g_{4}^{(n)}(,),
\end{aligned}
$$

where ${ }_{4 j}$ and $u_{4}$ are the electric displacement and the electric potential, respectively.

Determination of each intensity of singularity is obtained by calculated the stresses for each order of singularity as follows:

$$
{ }_{i j}(r, \quad, \quad)=K\left(\frac{r}{L}\right)^{-} f_{i j}(, \quad),
$$

The displacement field is of the following form:

$$
u_{i}(r, \quad, \quad)=K\left(\frac{r^{1}}{L}\right) g_{i}(, \quad) \text {. }
$$

The primed solution is a complementary solution with an order of singularity of $\lambda=3-\lambda$ (Lee and Im [6]). The stress components and the displacements are

$$
\begin{aligned}
& i_{i j}^{\prime}(r, \quad)=C\left(\frac{r}{L}\right)^{-} f_{i j}^{\prime}(,), \\
& u_{i}(r, \quad)=C\left(\frac{r^{1}}{L}\right) g_{i}^{\prime}(, \quad) .
\end{aligned}
$$

Finally, the intensity of singularity can be obtained from

$$
K=H={ }_{S}\left({ }_{i j} u_{i} \quad{ }_{i j} u_{i}\right) \hat{n}_{j} d s,
$$

where $S_{\Gamma}$ is an arbitrary surface around the singular point.

\section{Numerical Analysis}

\section{A. The Model for Analysis}

The model used for the analysis is illustrated in Fig. 1. The model is a bi-material bonded joint that consists of PZT-4 as an upper material and PZT-5H as a lower material. Mechanical loading, a uniform tensile stress $\left({ }_{0}=10 \mathrm{MPa}\right)$, is applied to the top surface. The model is fixed on the bottom surface. The length, $L$, is fixed at $5 \mathrm{~mm}$. Dimensions and boundary conditions are shown in Fig. 1, and the material properties are listed in Table 1.

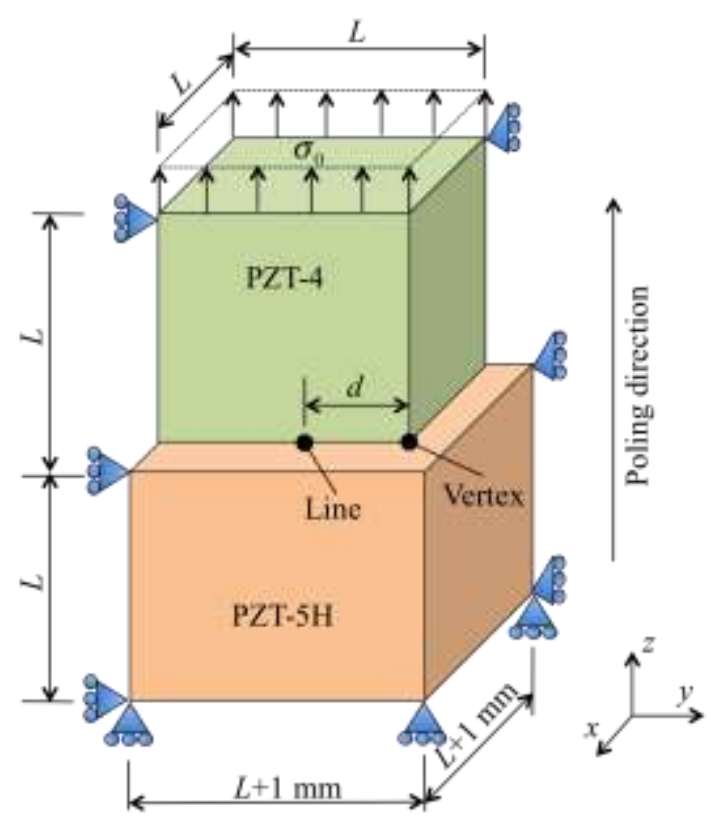

Fig. 1 Analytical model and boundary conditions

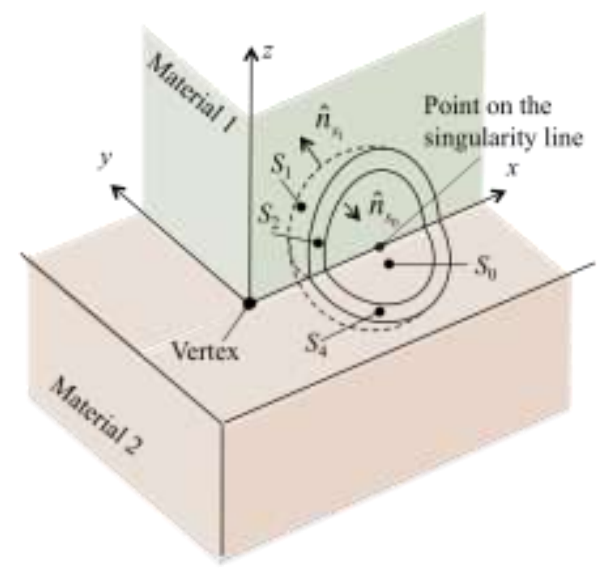

Fig. 2 A specific closed area for integral

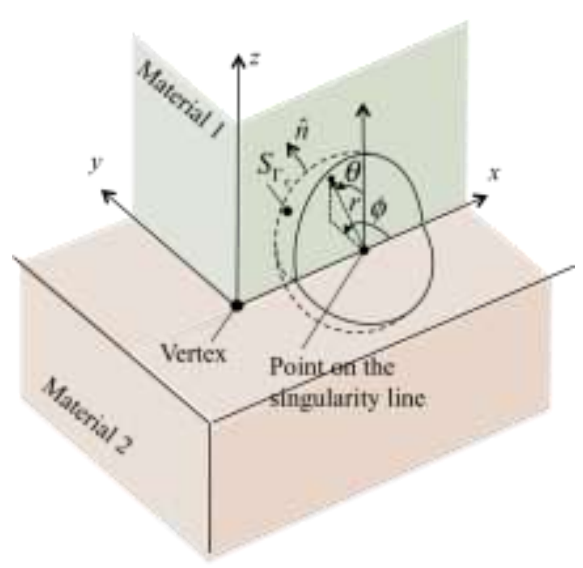

Fig. 3 A small circle area, $S_{\Gamma}$ 
Proc. of Sixth International Conference On Advances in Civil, Structural and Mechanical Engineering -ACSM 2017 Copyright (C) Institute of Research Engineers and Doctors, USA .All rights reserved.

ISBN: 978-1-63248-118-4 doi: 10.15224/ 978-1-63248-118-4-24

TABLE I. MATERIAL PROPERTIES

\begin{tabular}{|c|c|c|c|}
\hline & & PZT-4 & PZT-5H \\
\hline \multirow{4}{*}{ Elastic Constant, GPa } & $C_{11}$ & 139 & 126 \\
\cline { 2 - 4 } & $C_{12}$ & 77.8 & 55 \\
\cline { 2 - 4 } & $C_{13}$ & 74.3 & 53 \\
\cline { 2 - 4 } & $C_{33}$ & 113 & 117 \\
\cline { 2 - 4 } & $C_{44}$ & 25.6 & 35.3 \\
\hline \multirow{3}{*}{$\begin{array}{c}\text { Piezoelectric Constant, } \\
\text { C/m }\end{array}$} & $e_{31}$ & -6.98 & -6.5 \\
\cline { 2 - 4 } & $e_{33}$ & 13.8 & 23.3 \\
\cline { 2 - 4 } & $e_{15}$ & 13.4 & 27.0 \\
\hline \multirow{2}{*}{$\begin{array}{c}\text { Dielectric Constant, 10-10 } \\
\text { C/Vm }\end{array}$} & $\chi_{11}$ & 60.0 & 151 \\
\cline { 2 - 4 } & $\chi_{33}$ & 54.7 & 130 \\
\hline \multirow{2}{*}{\begin{tabular}{c} 
C/Vm \\
\hline
\end{tabular}}
\end{tabular}

TABLE II. THE ORDERS OF STRESS SINGULARITIES

\begin{tabular}{|c|c|c|}
\hline & $\lambda_{\text {vertex }}$ & $\lambda_{\text {line }}$ \\
\hline$(1)$ & 0.587 & 0.458 \\
\hline$(2)$ & 0.313 & 0.312 \\
\hline
\end{tabular}

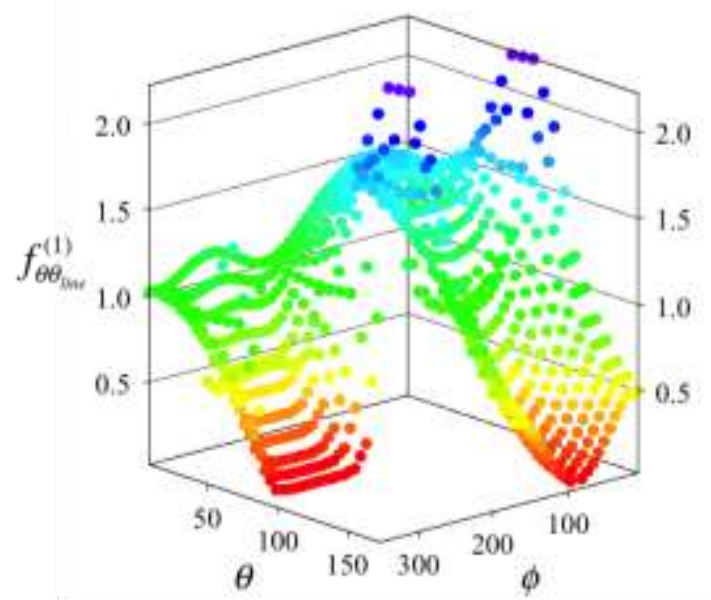

Fig. 4 Angular function of stresses; $f_{\theta \theta}^{\stackrel{(1)}{l i n e}(\theta, \phi)}$

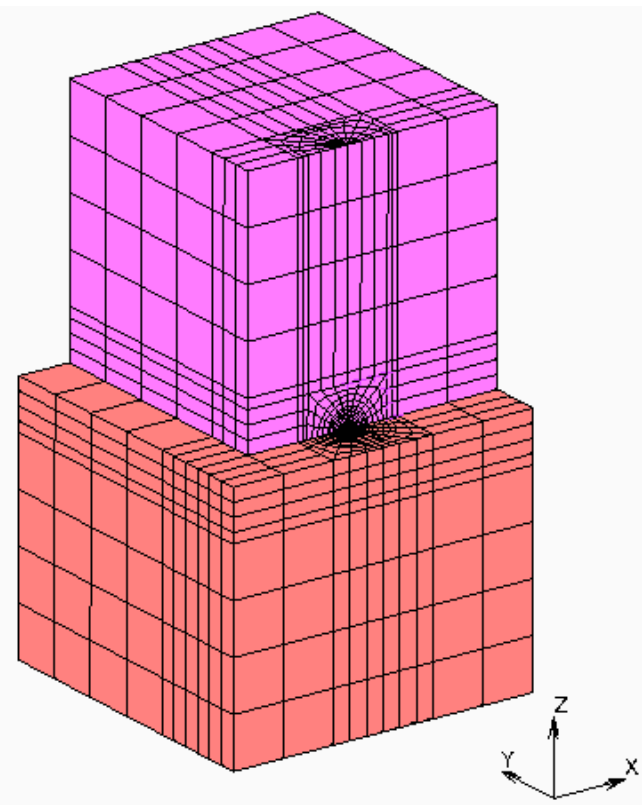

Fig. 5 Mesh model for FE analysis $(d=2 \mathrm{~mm})$

\section{B. Orders of Singularity and Angular Functions}

Eigenanalysis formulated by a three-dimensional FEM (Islam and Koguchi [4]) is used to calculate orders of stress singularity. The eigenequation derived by the principle of virtual work for calculating the eigenvalue, $p$, is expressed as follows:

$$
\left(p^{2}[\mathbf{A}]+p[\mathbf{B}]+[\mathbf{C}]\right)\{\mathbf{u}\}=0,
$$

where $[\mathbf{A}],[\mathbf{B}]$, and $[\mathbf{C}]$ are matrices composed of the material properties, $p=1-\lambda$, and $\{\mathbf{u}\}$ is the eigenvector of displacement (refer to Pageau and Bigger [5] for details).

The results for the orders of the singularity at the vertex, $\lambda_{\text {vertex }}$, and the orders of the singularity along the singular line, $\lambda_{\text {line }}$ are presented in Table 2 . This model consists of multi-term of singularities. In this study, we have considered two-major terms of singularities, and defined $\lambda^{(1)}$ to be the largest order of singularity. As shown in Table 2, the magnitude of $\lambda_{\text {vertex }}{ }^{(1)}$ is larger than that of $\lambda_{\text {line }}{ }^{(1)}$. However, the magnitude of $\lambda_{\text {vertex }}{ }^{(2)}$ and $\lambda_{\text {line }}{ }^{(2)}$ are nearly the same value.

After calculating the orders of the singularity, the angular functions of the displacements and the electric potential, $g_{i}(\theta, \phi)$ and $g_{i}^{\prime}(\theta, \phi)$, shown in Eqs. (7) and (9) are obtained using eigenvector analysis. The angular functions of the displacements and the electric potential are then converted to the angular functions of the stresses and the electric displacements, $f_{i j}(\theta, \phi)$ and $f^{\prime}{ }_{i j}(\theta, \phi)$, shown in Eqs. (6) and (8) following the stress-strain relation.

The angular functions at the vertex are normalized such that

$$
f_{\theta \theta \text { vertex }}^{(1),(2)}\left(\frac{\pi}{2}, \frac{\pi}{4}\right)=1, f_{\theta \theta_{\text {vertex }}^{\prime}}^{(1),(2)}\left(\frac{\pi}{2}, \frac{\pi}{4}\right)=1,
$$

Details of the eigenvalue, the eigenvector analysis and the angular functions at the vertex are shown in Luangarpa and Koguchi [3].

Next, the angular functions for the point on the singular line are normalized such that

$$
f_{\theta \theta \text { line }}^{(1),(2)}\left(\frac{\pi}{2}, \frac{\pi}{2}\right)=1, f_{\theta \theta \text { line }}^{\prime}(1),(2)\left(\frac{\pi}{2}, \frac{\pi}{2}\right)=1 .
$$

For instance, the angular function of stress, $f_{\theta \theta}^{\stackrel{(1)}{\text { line }}}(\theta, \phi)$, is shown in Fig. 4.

\section{Intensities of Singularity at the vertex}

The conservative integral was used to analyse the intensity of the singularity at the vertex. The singular solutions used in Eq. (14) were obtained using the Marc2015 finite element (FE) program. Eight-node elements are employed. The elements around the vertex are organized to be spherical in shape and centred at the vertex. The angular dimensions of the element are $=15^{\circ}$ and $\theta=15^{\circ}$. The size of the smallest element connected to the singular point is $0.01 \mathrm{~mm}$ in the $r$-direction. The integral surface area is the area with a distance $0.05 \mathrm{~mm}$ from the vertex.

The results of the intensity of the singularity are; $K_{\text {vertex }}{ }^{(1)}$ $=1.05$ and $K_{\text {vertex }}{ }^{(2)}=0.96$. (Details of the conservative 
Proc. of Sixth International Conference On Advances in Civil, Structural and Mechanical Engineering -ACSM 2017 Copyright (c) Institute of Research Engineers and Doctors, USA .All rights reserved.

ISBN: 978-1-63248-118-4 doi: 10.15224/ 978-1-63248-118-4-24

integral for the vertex are presented in Luangarpa and Koguchi [3].)

\section{Intensities of Singularities along the singular line}

The conservative integral is extended to obtain the intensity of the singularity at a point on the stress singular line. Example of the element model used in the FE analysis is shown in Fig. 5 (the model with $d=2 \mathrm{~mm}$ ). The eightnode element is used in the same manner as in the analysis at the vertex. The elements around the singular point are organized to be spherical shape. Similar to the analysis at the vertex, the angular dimensions of the element are $\phi=$ $15^{\circ}$ and $\theta=15^{\circ}$; and the size of the smallest element connected to the singular point is $0.01 \mathrm{~mm}$ in the $r$-direction. The integral surface area is the area with a distance $0.05 \mathrm{~mm}$ from the investigated point.

Fig. 6 shows the variations of $K_{\text {line }}^{(1)}$ and $K_{\text {line }}^{(2)}$ with $\left(\frac{d}{L}\right)$, where $d$ is the distance from the vertex. Both $K_{\text {line }}^{(1)}$ and $K_{\text {line }}^{(2)}$ approach constant values at a point further from the vertex. The values of $K_{\text {line }}^{(1)}$ are much larger than that of $K_{\text {line }}^{(2)}$. That means we may predict the singular fields along the singular line by considering the first-term of singularity. In addition, $K_{\text {line }}^{(1)}$ increases greatly as the vertex approaches. Fig. 7 shows the distributions of stresses, line, at $=90^{\circ}$ and $\theta=90^{\circ}$ with respect to $r$ for $d=0.1,0.5$ and $1.0 \mathrm{~mm}$. This figure also indicated that the magnitude of stress increases at the point closed to the vertex, which means that the singularity at the vertex may affect the singularity along the singularity line.

\section{Conclusions}

The conservative integral based on the Betti reciprocal principle was developed to calculate the intensity of singularity along the singular line in three-dimensional piezoelectric-material joints with two-real singularities. The results for the intensities of singularities along the singular line revealed that $K_{\text {line }}^{(1)}$ increased as the vertex approaches, while $K_{\text {line }}^{(2)}$ was stable. Relationship between the singularity at the vertex and along the singular line should be considered.

\section{References}

[1] C. Luangarpa, and H. Koguchi, "Analysis of a three-dimensional dissimilar material joint with one real singularity using a conservative integral,” Int. J. Solid Struct, vol. 51, pp. 2908-2919, 2014.

[2] C. Luangarpa, and H. Koguchi, "Analysis of singular stresses at a vertex and along a singular line in three-dimensional bonded joints using a conservative integral," Euro. J. Mech. A/Solids, vol. 60, pp. 208-216, 2016.

[3] C. Luangarpa, and H. Koguchi, "Analysis of singular stress fields at 3D piezoelectric bonded joints using a conservative integral," ASME International Mechanical Engineering Congress and Exposition, Proceedings (IMECE), 2016.
[4] M.S. Islam, and H. Koguchi, "Characteristics of Singular Stress Distribution at a Vertex in Transversely Isotropic Piezoelectric Dissimilar Material Joints,” J. Solid Mech. and Mat. Eng., vol. 4, no.7, pp.1011-1026, 2010.

[5] S.S. Pageau, and Jr S. B. Biggers, "Finite Element Evaluation of FreeEdge Singular Stress Fields in Anisotropic Materials," Int. J. for Numerical Methods in Eng., vol. 38, pp. 2225-2239, 1995.

[6] Y. Lee, S. Im, "On the computation of the near-tip stress intensities for three-dimensional wedges via two-state M-integral," J. Mech. Phys. Solids., vol. 51, pp. 825-850, 2003.

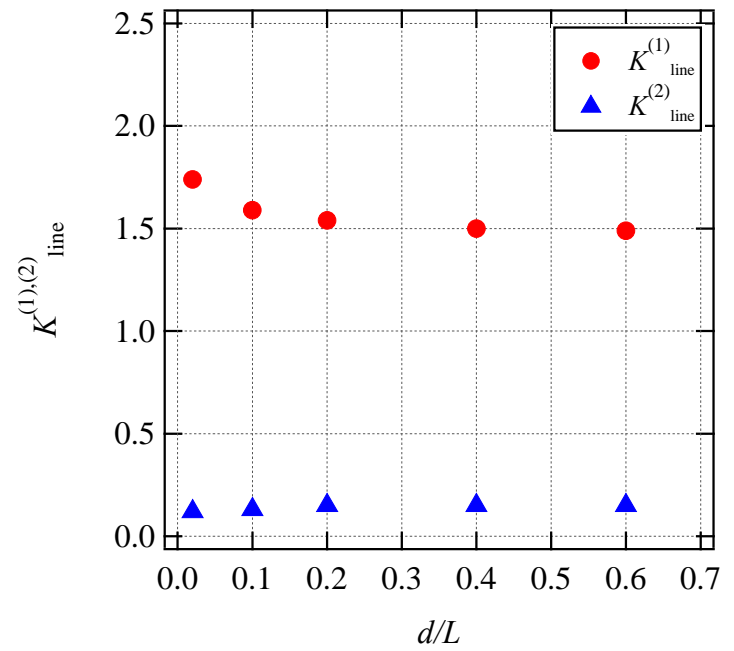

Fig. 6 The intensity of singularity on a singular line with $d / L$

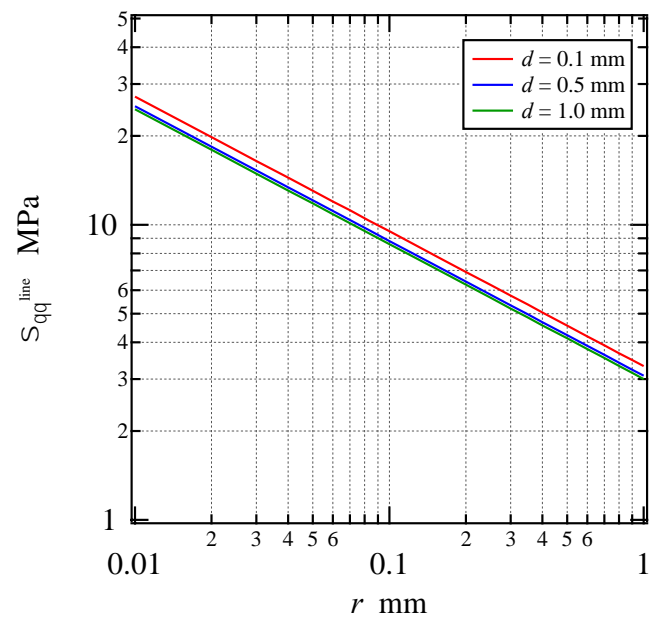

Fig. 7 Distribution of stresses, line , at $\quad=90^{\circ}$ and $\theta=90^{\circ}$ with $r$.

About Author (s):

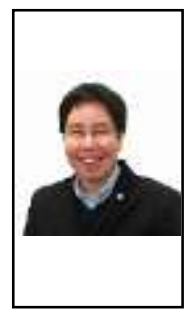

Dr. Chonlada Luangarpa earned her doctor of engineering from Nagaoka University of Technology, Japan. She is currently an assistant professor of computational mechanics at Department of Mechanical Engineering, Nagaoka University of Technology.

Dr. Hideo Koguchi is a professor of computational mechanics at Mechanical Engineering Department. He is also the dean of Mechanical Engineering Department, Nagaoka University of Technology.

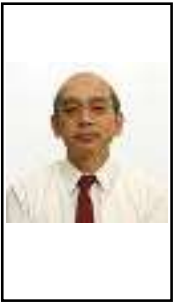

